Nachhaltiger Konsum

\section{Der SustainCoin als Nachhaltigkeitslabel}

\author{
Nachhaltigkeits-Label versetzen potenzielle Kunden in die Lage, \\ eine Kaufentscheidung von für sie wichtigen Kriterien abhängig \\ zu machen. Der SustainCoin stellt ein neues Label dar, das Natur- \\ verbrauch, Soziallasten und Gesamtkosten grafisch darstellt. \\ Von Klaus Hildenbrand
}

B ereits seit dem April dieses Jahres hat die Bundesrepublik Deutschland das Budget an Treibhausgasemissionen ausgeschöpft, welches nach ihrer Selbstverpflichtung aus dem Klimaschutzabkommen von Paris vom 12.12. 2015 für das gesamte Jahr 2017 anvisiert war. $\mathrm{Zu}$ dieser Erkenntnis gelangt Timm Kehler, der Vorsitzende der Brancheninitiative Erdgas unter Berufung auf eine Studie der Berliner Nymoen-Strategieberatung (tageschau24, 2017).

\section{Was Marktwirtschaft leisten kann}

Eine derartige Behauptung ist geeignet, die ohnehin bereits weitverbreitete Politikverdrossenheit noch $\mathrm{zu}$ verstärken. Sind die Politiker nicht gewillt oder nicht in der Lage, Ziele, für die sie sich einsetzen, auch umzusetzen? Dies wirft die grundsätzliche Frage auf, welche Rolle die Politik in einer möglichst frei agierenden Marktwirtschaft übernehmen kann. In der westlichen Welt besteht Konsens darüber, dass sie einen Rahmen definieren muss, innerhalb dessen die Wirtschaft dann aber mit den maximal möglichen Freiheiten selbstständig agieren können muss.

Ist diese Vorstellung der nur ordnenden Funktion der Politik anlässlich der sich zuspitzenden Klimakatastrophe und den sich daraus ergebenden sozialen Verwerfungen noch zeitgemäß oder müssten mit einer stärker regulierten Wirtschaft die notwendigen Reduktionsziele erzwungen werden?
Eine derartige Wirtschaftspolitik würde an den Grundfesten unseres Demokratie- und Wirtschaftsverständnisses rütteln und ihre Wirksamkeit wäre zudem fraglich.

Wie ist es also möglich, Freiheitsrechte $\mathrm{zu}$ bewahren und trotzdem $\mathrm{zu}$ wesentlich deutlicheren Reduktionszielen zu gelangen als den bisherigen?

Maximale Freiheit der Vertragspartner in Wirtschaftsprozessen in Verbindung mit verantwortungsvollem Handeln sind nur möglich, wenn die Informationsasymmetrien der Vertragsparteien aufgehoben werden. Solange es Konsumenten nicht möglich ist $\mathrm{zu}$ erkennen, welche Auswirkungen ihre Kaufentscheidungen haben, sind sie nicht in der Lage, zukunftsweisende Entscheidungen zu treffen.

Label als Qualitätssiegel sollen hier derzeit Abhilfe schaffen, deren Wirksamkeit sind indes beschränkt. Aktuell wird beispielsweise ein sogenanntes Tierwohllabel diskutiert - welche tatsächlich positiven Auswirkungen dies aber auf die Schweinezucht haben wird, ist noch unklar. Erreicht man beispielsweise mit der Vergrößerung der Stellfläche um $0,25 \mathrm{~m}^{2} /$ Schwein tatsächlich bemerkenswert bessere Bedingungen in der Schweinezucht? Wie kann sich ein Landwirt in einem solchen System profilieren, der es beispielsweise für richtig erachtet, seinen Schweinen eine Erhöhung der Stellfläche um $2 \mathrm{~m}^{2}$ gegenüber der normierten Mindestfläche zu gewähren?

Unabhängig, wie fragwürdig Kriterien für derartige Labels sind, allen ge- mein ist, dass sie wenig Dynamik aufweisen und sich daher nicht schnell genug an neue Anforderungen anpassen können. Wäre beispielsweise ein mit einem Label beworbenes Produkt hinsichtlich seiner ökosozialen Performance nicht mehr empfehlenswert, wird man derzeit diesen Hinweis sicher auf keinem dieser Label vorfinden.

Um aber noch eine reelle Chance $\mathrm{zu}$ haben, unsere globalen Umwelt- und Sozialziele erreichen zu können, ist es unabdingbar, ein dynamisches, lernendes System zu installieren, das bei Kaufentscheidungen Anreize bietet, Produkten und Dienstleistungen mit der jeweils besten ökosozialen Performance den Vorzug zu geben.

\section{Der SustainCoin}

Der SustainCoin will hierzu seinen Beitrag leisten, indem er den Konsument zum Zeitpunkt seiner Kaufentscheidung in die Lage versetzt, auch als Laie komplexe ökosoziale Folgen seiner Kaufentscheidung beurteilen zu können.

Gegliedert in die Bereiche Umwelt, Soziales, Finanzen, Funktion und Prozess und angebracht auf Preisschildern oder eingefügt in Angebotsunterlagen liefert er Informationen zum Naturverbrauch, zu den Kosten vom Kauf bis zur Entsorgung, zum Gerechtigkeitsindikator entlang der gesamten Wertschöpfungskette, der durch CE-Leistungserklärungen dokumentierten Funktionen und schließlich zum Operationalisierungsgrad der innerbetrieblichen Prozesse.

Das obere Feld des SustainCoins kann braun oder grün markiert sein. Auf diese Weise kann der Naturverbrauch (braun) gegenüber beispielsweise Naturgewinn (grün) einprägsam visualisiert werden. Je nach Füllung der Ringe wird deutlich, wie stark die jeweilige Eigenschaft ausgeprägt ist.

Um die farblich abgesetzte Schraffur in der richtigen Dimension einordnen zu können, gibt der Exponent am Rand des SustainCoins an, welchen Wert dieser Sektor annimmt, wenn er über den gesamten Bereich der zehn Ringe schraf- 


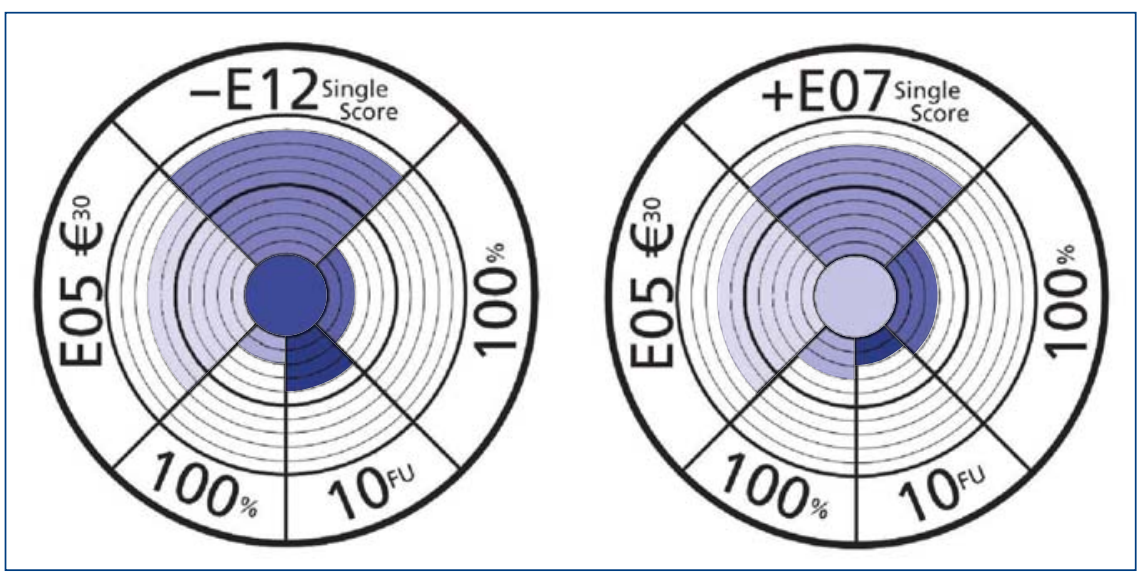

Abbildung 1: Vergleich SustainCoin mit Naturverbrauch (links) und Naturgewinn (rechts). (Quelle: hilbra)

fiert ist. Mit dem negativen Vorzeichen wird der Naturverbrauch beschrieben, während der positive Betrag, wie er am rechten Beispiel dargestellt ist, einen Naturgewinn darstellt (zum Beispiel: Produktion von biologisch angebautem Gemüse).

Als Einheit dient der Single Score. Es handelt sich hierbei um das Ergebnis der Aggregierung der Ressourcenindikatoren, welche in einem mehrstufigen Bewertungsprozess nach der ReCiPeMethode aus den Daten der Datenbank ecoinvent mit der Open Source Software Open LCA (Ciroth/Franze 2011) gewonnen wird.

Betrachtet man nun die beiden Beispiele aus Abbildung 1, welche zwei völlig unterschiedliche Produkte klassifizieren, so zeigt sich, dass mithilfe der Exponentialform ein Zahlenraum von $-10^{99}$ bis $10^{99}$ dargestellt werden kann. Auf diese Weise ist es problemlos möglich, sowohl den Naturverbrauch für ein Kernkraftwerk als auch den Naturgewinn durch den Anbau einer biologischen Karotte darzustellen.

Der am Rand angegebene Wert gibt den Maßstab an, in dem die darunterliegende Schraffur eingeordnet werden kann. So zeigt beispielsweise der rechte Coin einen Single Score von $+8 \times 10^{6}$.

Verwendet man beispielsweise den SustainCoin zur Einordnung zweier unterschiedlicher Automodelle, so ist es leicht, die beiden Modelle anhand der "Befüllung“ des entsprechenden Sektors mit Schraffur zu bewerten, wenn sich die beiden Naturverbräuche innerhalb einer Zehnerpotenz bewegen. Sollten sich die beiden Naturverbräuche jedoch in zwei unterschiedlichen Zehnerpotenzen bewegen, so ist neben der „Befüllung" des Sektors auch dessen Maßstab in Form der abweichenden Zehnerpotenz zu bewerten.

Während man bei Vergleichen innerhalb einer Branche also durch die grafische Darstellung bei der Beurteilung des Single Scores grafisch unterstützt wird, hat man durch den weiten zur Verfügung stehenden Zahlenraum die Möglichkeit, jedes $\mathrm{x}$-beliebige Produkt und jede $\mathrm{x}$-beliebige Dienstleistung darzustellen.

\section{Bereiche der Bewertung}

Das rote Feld beschreibt den sozialen Aspekt der Einkommensverteilung. Ausgehend von der Überzeugung, dass diese einen ganz wesentlichen Indikator für die Arbeitsbedingungen innerhalb eines Wertschöpfungsprozesses darstellt, wird diese trotz des Einflusses sozialer Aspekte auf den Single Score hier zusätzlich und gleichberechtigt betrachtet. Hat beispielsweise ein Minenarbeiter ein so geringes Einkommen, dass er nur unter schwierigsten Bedingungen sich und seine Familie ernähren kann, wird er nicht mit hohem Selbstbewusstsein seine Arbeitnehmerrechte durchsetzen können.

Grundlage der Berechnung ist das Gerechtigkeitsmodell, das auf einer Lorenzkurve basiert, welche mit einem

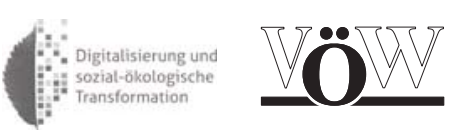

Fishbowl Diskussion

mit Tilman Santarius (TU Berlin), Laura Dornheim (Grüne), Leon Reiner (Impact Hub) und den Teilnehmenden der VÖW Herbstakademie Moderation: Anne Fromm taz

Sonntag, 8. Oktober 2017 14:30 - 16:30 Uhr

Impact Hub Berlin,

Friedrichstrasse 246

www.voew.de/herbsakademie www.nachhaltige-digitalisierung.de Anmeldung: info@voew.de

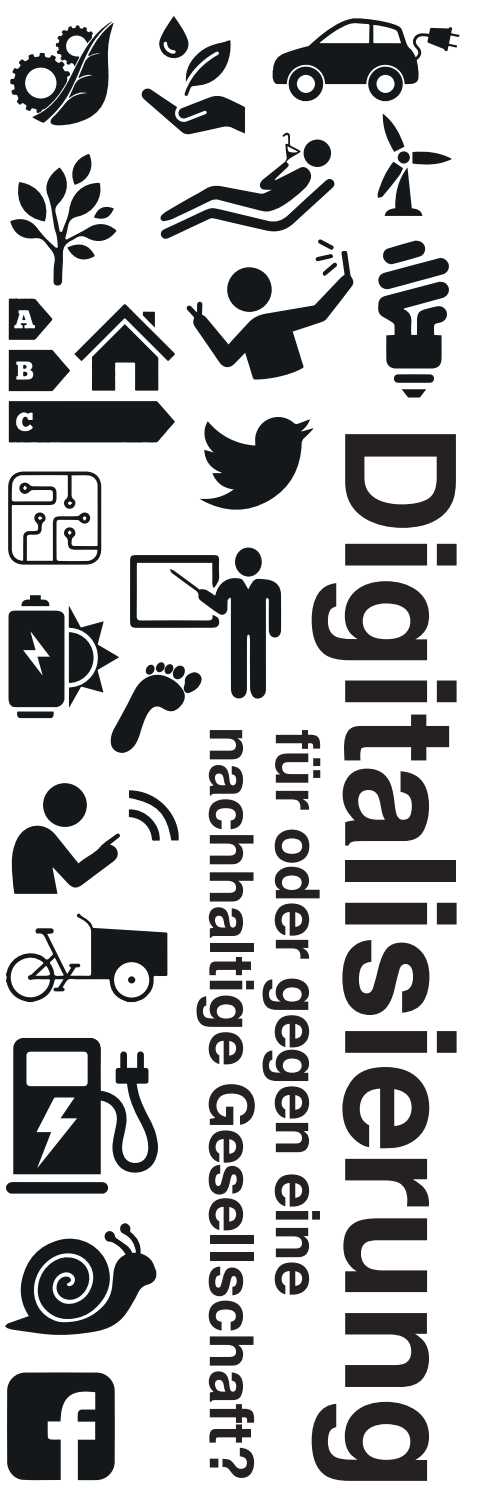


Equity-Faktor von 0,618 als Gerechtigkeitsideal und somit als Referenz für die Bewertung der tatsächlichen Einkommensverteilung herangezogen wird (Hildenbrand 2013). Um nun einen Wert zu erhalten, der für die Beurteilung des betrachteten Produktes von Bedeutung ist, werden die einzelnen Equity-Faktoren aus den die Wertschöpfungskette berührenden Unternehmen mit den anteiligen Zeitfaktoren gewichtet, um den GesamtEquity-Faktor zu erhalten.

Eines der wichtigsten Kriterien beim Kauf eines Produkts ist derzeit noch der Preis. Dieser kann aber, auch aus rein wirtschaftlicher Sicht, nur zuverlässig bewertet werden, wenn der potenzielle Käufer neben dem Einstandspreis auch Kenntnis darüber erhält, welche Kosten zum Beispiel für Wartung und Reparatur für die nächsten $\mathrm{x}$-Jahre (abhängig von der Branche) kalkuliert werden müssen. Angegeben in diesem Sektor sind daher die Barkosten, welche auch die entsprechend abdiskontierten Folgekosten mitberücksichtigen. Die Darstellung des Betrags folgt hierbei der Systematik, wie sie bereits im Sektor Umwelt beschrieben wurde.

Der Bereich Technik, der im blauen Feld beschrieben wird, hat nur eine unterstützende Funktion. Er soll die Kaufentscheidung in einen bewertbaren Kontext stellen. Hier wird die Anzahl der Funktionen angegeben, die zum Beispiel durch einen erfolgreich durchgeführten CE-Prozess dokumentiert und für das entsprechende Produkt garantiert sind.

Beispielsweise können Horizontalfaltläden auch als Sonnenschutzelemente verwendet werden. Dass sie hierfür auch geeignet sind, wird im Rahmen des CE-Prozesses unter Angabe der als Grundlage dienenden Normen garantiert. Wird auf gleichem Weg beispielsweise zusätzlich die Funktion als Brandschutz garantiert, könnte eventuell auf einen sonst zusätzlich zu montierenden Brandschutzrollladen und somit auch auf dessen zusätzlichen Naturverbrauch verzichtet werden.

Ebenso wie der Bereich Technik dient der Sektor Prozess und somit das orangenfarbene Feld des SustainCoins dem
Ziel, die drei Nachhaltigkeitskriterien im entsprechenden Kontext zu sehen. Mit dem Grad der „Befüllung“ dieses Sektors wird der Operationalisierungsgrad visualisiert. Dieser sagt aus, wie weit die Prozesse bereits operationalisiert sind und welche notwendig sind, um die oben genannten Bereiche vollumfänglich bewerten zu können. Bisher konzentrierte sich die Forschungsarbeit auf die Entwicklung der Infrastruktur des SustainCoins, erst mit der Akzeptanz dieses Systems kann in die Steigerung des Operationalisierungsgrades investiert werden. Deshalb ist er in den beiden Beispielen aus Abbildung 1 noch recht niedrig dargestellt.

\section{Diskussion}

Die Betrachtung der Prozesse, welche zum SustainCoin führen, macht deutlich, dass der Informationsgehalt zwar sehr hoch und sicher auch sehr zielführend ist, er wird jedoch durch einen nicht minder hohen administrativen Arbeitsaufwand erkauft. Führt ein Unternehmen ein solches System ein, könnte es aufgrund dieser zusätzlichen Kosten Schwierigkeiten am Markt bekommen, welche aus dem notwendigerweise höheren Preis gegenüber dem Wettbewerb resultieren könnte. Dieser Nachteil könnte jedoch durch die Entwicklung entsprechender Erweiterungen für ERP-Systeme weitestgehend kompensiert werden.

Ein eindeutiger Vorteil der Einführung eines Systems, wie der SustainCoin es darstellt, ist die Möglichkeit, sich hierdurch ein Alleinstellungsmerkmal zu erarbeiten. Diese Möglichkeit wird nach Überzeugung des Autors den oben genannten Nachteil ganz deutlich überwiegen.

\section{Ausblick}

Mit der Einführung des SustainCoins im Unternehmen und dessen Ausweitung auf das gesamte Produktportfolio bietet sich die Möglichkeit, auch die oben beschriebenen Nachhaltigkeitsaspekte zu bilanzieren. Dies böte in $\mathrm{Zu}$ kunft die Chance, entsprechend agie- rende Unternehmen steuerrechtlich $\mathrm{zu}$ unterstützen und die nachhaltige Entwicklung wirtschaftlich attraktiver $\mathrm{zu}$ gestalten.

Die rasant fortschreitende Digitalisierung in den Industriestaaten bietet die Möglichkeit, das SustainCoin-System einzuführen, ohne dass das operative Geschäft dadurch wesentlich beeinträchtigt würde. Sieht man beispielsweise in der Baubranche den zunehmenden Trend zum integralen Planen, welches derzeit unter dem Schlagwort BIM diskutiert wird, kann man erkennen, dass allerorten die Zeichen auf Transparenz stehen, wie sie der SustainCoin bieten kann.

\section{Anmerkung}

In Kooperation mit der Dualen Hochschule Baden-Württemberg und Unternehmen aus den unterschiedlichsten Branchen plant der Autor derzeit das Forschungsprogramm COIN. Hierbei soll der Frage nachgegangen werden, inwieweit das Integrierte Managementsystem SNE (Hildenbrand 2013), das den Weg der Datenaggregation bis zur Erstellung des SustainCoins beschreibt, von den beteiligten Unternehmen angewandt werden kann. Ferner soll der Anspruch, mithilfe des SustainCoins Nachhaltigkeitskriterien von beliebigen Produkten und Dienstleistungen darstellen zu können, verifiziert werden.

\section{Literatur \\ tagesschau24 (2017): $\mathrm{CO}_{2}$-Jahresbudget schon aufgebraucht. Im Internet unter: https://www.tagesschau.de/inland/ deutschland-emissionen-101.html (letzter Aufruf: 10. April 2017) \\ Ciroth, A./Franze, J. (2011): LCA of an Ecolabeled Notebook. \\ Hildenbrand, K. (2013): Der Weg zur nach- haltigen Kaufentscheidung - Ein Lösungs- ansatz der hilbra Theo Hildenbrand $\mathrm{GmbH}$.}

AUTOR + KONTAKT

Klaus Hildenbrand ist Wirtschaftsingenieur Tischlermeister und Geschäftsführer der hilbra Theo Hildenbrand $\mathrm{GmbH}$.

hilbra Theo Hildenbrand $\mathrm{GmbH}$, Finkenstr. 8, 97896 Freudenberg-Ebenheid. Tel.: +499378383, E-Mail: klaus.hildenbrand@hilbra.de 